meal for males and a 650 calorie meal for females, and are divided into sections for predetermined volumes of carbohydrates, proteins, cheese and sauces, the remainder of the plate being for vegetables. The bowl is designed to allow cereal and half a cup of milk. The control group received their usual clinical care. Compared with the control group, patients in the intervention group lost significantly more weight, had a greater decrease in non-HDL cholesterol and a greater change in systolic blood pressure. A significantly greater proportion of patients experienced a decrease in hypoglycemic medication and achieved at least $5 \%$ weight loss. Unlike patients taking insulin in the control group, patients on insulin in the intervention group had lost weight. The authors conclude that the portion-control plate is an inexpensive and effective tool to induce weight loss and decrease hypoglycemic medication in obese patients with type 2 diabetes. It could also be useful in overweight, nondiabetic subjects.

Original article Pedersen SD et al. (2007) Portion control plate for weight loss in obese patients with type 2 diabetes mellitus: a controlled clinical trial. Arch Intern Med 167: 1277-1283

\section{Simvastatin reduces biomarkers of inflammation in patients with type 1 diabetes mellitus}

Coronary artery disease is a major cause of death in patients with type 1 diabetes, and these patients have been reported to be in a proinflammatory state. Inflammation is an important factor in the pathogenesis of atherosclerosis. Anti-atherogenic properties, independent of their cholesterol-lowering effect, have recently been attributed to statins.

Jialal et al. conducted a randomized, placebo controlled, double-blind trial to examine the effects of simvastatin therapy on biomarkers of inflammation in young patients (mean age 23.4 years) with type 1 diabetes. Patients $(n=52)$ were assigned to placebo or simvastatin $20 \mathrm{mg} /$ day for 3 months.

Compared with baseline and placebo, simvastatin significantly reduced total serum cholesterol, LDL cholesterol and non-HDL cholesterol. Plasma levels of c-reactive protein and interleukin 8 (IL-8) were also significantly reduced, as was monocyte nuclear factor-kappa B activity. Release of superoxide, IL-8 and tumor necrosis factor from lipopolysaccharide-stimulated monocytes was also significantly reduced by simvastatin. The drug did not result in any significant changes in serum $\mathrm{HbA}_{1 \mathrm{c}}$ levels.

The authors conclude that statins seem to have a beneficial effect on the lipid profile as well as on inflammatory biomarkers and suggest that these findings could have implications for the development of policy guidelines for statin therapy in young patients with type 1 diabetes.

Original article Jialal I et al. (2007) Concomitant reduction of low-density lipoprotein-cholesterol and biomarkers of inflammation with low-dose simvastatin therapy in patients with type 1 diabetes. J Clin Endocrinol Metab 92:3136-3140

\section{Long-term effects of somatostatin analogs in patients with acromegaly}

Acromegaly is due to hypersecretion of growth hormone and insulin-like growth factor I (IGF-I). The aim of treatment is to limit this excess secretion and to reduce morbidity and mortality. Medical treatment of the disease is usually with somatostatin analogs.

An open, prospective, single-center study by Maiza et al. examined the long term ( $\leq 18$ years) antihormonal and antitumor efficacy as well as the safety of somatostatin analogs in 36 patients (mean age 53 years) with acromegaly. Patients received somatostatin analogs as primary therapy. They were assessed clinically, biologically and radiologically at the time of diagnosis, 6 months after starting treatment and then annually.

Growth hormone levels decreased from $13.5 \pm 3.1 \mu \mathrm{g} / \mathrm{l}$ pretreatment to $3.8 \pm 0.8 \mu \mathrm{g} / \mathrm{l}$ after 6 months and $1.6 \pm 0.1 \mu \mathrm{g} / \mathrm{l}$ after 10 years. IGF-I levels, relative to the normal range, decreased from $302 \pm 26 \%$ pretreatment to $181 \pm 14 \%$ after 6 months and $124 \pm 18 \%$ after 10 years. In 21 out of 29 patients analyzed, more than $20 \%$ tumor shrinkage was observed. Mean reduction in tumor size at the last measurement was $43 \%$. Although blood glucose levels deteriorated in 8 patients, none of the patients included had to stop primary medical therapy as a result of gastrointestinal, cutaneous or metabolic side effects. The authors concluded that long-term treatment with somatostatin analogs is effective and well tolerated.

Original article Maiza JC et al. (2007) Long-term (up to 18 years) effects on GH/IGF-1 hypersecretion and tumour size of primary somatostatin analogue (SSTa) therapy in patients with $\mathrm{GH}$-secreting pituitary adenoma responsive to SSTa. Clin Endocrinol (Oxf) 67: 282-289 